\title{
Role of diffusion-weighted magnetic resonance imaging in the evaluation of vertebral bone marrow lesions
}

\author{
Arashdeep Kaur ${ }^{1 A, B, C, D, E, F}$, Chuni L. Thukral| ${ }^{2 A, B, D}$, Gagan Khanna ${ }^{3 A, B, D}$, Paramdeep Singh ${ }^{1 C, D, E, F}$ \\ 'Department of Radiology, Guru Gobind Singh (GGS) Medical College and Hospital, Baba Farid University of Health Sciences, India \\ 2Department of Radiodiagnosis and Imaging, Sri Guru Ram Das Institute of Medical Sciences, India \\ ${ }^{3}$ Department of Orthopaedics, Sri Guru Ram Das Institute of Medical Sciences
}

\section{Abstract}

Purpose: To evaluate the role of diffusion-weighted magnetic resonance imaging (DW-MRI) in differentiating vertebral marrow pathologies. To determine the sensitivity, specificity, and threshold apparent diffusion coefficient (ADC) values that can aid in the differentiation of malignant from benign bone marrow lesions.

Material and methods: This observational study included 100 patients, who underwent MRI examination with a 1.5 Tesla scanner. The ADC values of normal and pathological vertebrae were estimated, and the threshold ADC values were computed by receiver operating characteristic (ROC) analysis. The results were correlated with histopathological diagnosis, clinical follow-up, and other investigations. Statistical analysis was done by employing unpaired two-tailed Student's $t$-test and the $p$-value of $<0.05$ was deemed as statistically significant.

Results: Vertebral bone marrow lesions had a male predominance and there was a predilection towards thoracic and lumbar vertebrae, with L4 being the commonest. Metastasis was the commonest lesion, followed by spondylodiscitis. The mean ADC value of benign pathologies was significantly greater than malignant pathologies $(p<0.05)$. The threshold value for the demarcation between benign and malignant pathologies was computed to be $1.21 \times 10^{-3} \mathrm{~mm}^{2} / \mathrm{s}$. DW imaging had sensitivity of $100 \%$, specificity of $92.31 \%$, positive predictive value of $87.5 \%$, and negative predictive value of $100 \%$.

Conclusions: Vertebral marrow lesions can be differentiated as benign or malignant with good sensitivity and specificity with the help of DW-ADC maps.

Key words: metastasis, bone marrow, ADC, vertebrae, diffusion MRI, DWI.

\section{Introduction}

Vertebral bone marrow lesions are common, and imaging findings are associated with various aetiologies, such as degenerative, infectious, traumatic, malignant, metabolic, and haematopoietic; these lesions often have a non-specific appearance on magnetic resonance imaging (MRI) [1]. As compared to conventional radiography and computed tomography (CT), MRI is a better non-invasive imaging modality to evaluate vertebral bone marrow, due to better soft-tissue contrast as well as being non-ionising in nature $[1,2]$. At birth, the whole spinal marrow is metabolically active (haematopoietic/red marrow), and it gradually turns into a less metabolically active (fat/yellow) marrow with age. This temporal physiologic phenomenon is known as normal marrow conversion and concludes around the age of 25-30 years [1,2].

Routine evaluation of the spinal marrow comprises turbo spin-echo T1 W and T2 W pulse sequences along with STIR sequences. Contrast administration, although

Correspondence address:

Dr. Paramdeep Singh, Department of Radiology, Guru Gobind Singh (GGS) Medical College and Hospital, Baba Farid University of Health Sciences, India, e-mail: paramdeepdoctor@gmail.com

Authors' contribution:

A Study design · B Data collection · C Statistical analysis · D Data interpretation · E Manuscript preparation · F Literature search · G Funds collection 
leading to improvement of sensitivity and specificity of MRI studies, requires intravenous access and may be contraindicated in patients with deranged renal function, owing to the risk of nephrogenic systemic fibrosis (NSF) $[2,3]$. In our experience, routine MRI sequences are often insufficient in distinguishing malignant from benign vertebral bone marrow lesions, due to overlapping imaging features and often due to coexistence of bone marrow oedema that can be caused by fractures, infection, and bone marrow hyperplasia, thus reducing the specificity of conventional sequences.

Diffusion-weighted imaging (DWI) is a functional imaging sequence that reproduces the variances in the Brownian motion in water owing to dissimilarities in tissue microstructure and can be acquired without contrast. On the other hand, apparent diffusion coefficient (ADC) values provide a quantitative measure of Brownian movement with low ADC values implying a restricted diffusion and high values being a measure of free diffusion [4]. Therefore, DWI provides both qualitative and quantitative functional information concerning the microscopic movements of water at the cellular level. The benefits of DWI comprise a small scanning period that allows it to be easily included in the routine imaging protocols [5]. Some studies have suggested a significant role of DWI in the assessment of vertebral marrow pathologies while few others have shown only equivocal results [6]. We hypothesise that DW-ADC maps aid in the distinction between malignant and benign vertebral pathologies. Hence, we carried out this study in our set up to evaluate the role of DW-ADC maps in segregating benign and malignant vertebral marrow pathologies.

\section{Material and methods}

This observational research was done from October 2016 to October 2018 and consisted of 100 patients ( 40 females, 60 males) with a mean age of 53.7 years (range; 7 to 88 years).
Detailed clinical history along with physical examination was done and documented in all the patients. The inclusion criteria comprised age more than five years, history of back pain less than two months duration, bony pathology seen on X-ray radiographs, and bone marrow oedema at the fracture sites. The exclusion criteria included pregnancy and contraindications to MRI or contrast agents.

A total of 302 vertebral lesions were classified according to radiological findings into three groups, namely: benign, infectious, and malignant. The Institutional Ethical Committee approved this study, and written, informed consent was acquired from each of the patients.

All patients underwent MRI examinations with a Philips Gyroscan Achieva 1.5 Tesla MRI (Philips Healthcare, Best, Netherlands) using a Sense Body Coil. The following sequences were done: T1 WI (sagittal), T2 WI (sagittal and axial), STIR (sagittal), and DWI (sagittal). Fat-suppressed, contrast-enhanced, T1-weighted, and additional sequences were also used wherever required (Table 1). The pulse sequence employed in acquiring the DW images was a single shot echo-planar imaging (EPI) method with b values of $0,350 / 400$, and $700 / 800 \mathrm{~s} / \mathrm{mm}^{2}$ in the sagittal plane in all three directions $(\mathrm{x}, \mathrm{y}, \mathrm{z})$. A fat-saturated pulse was used in all images to eliminate chemical shift artefacts. Isotropic images were obtained by calculating the projection of diffusion vectors in three directions to every voxel for $a b$ value of $800 \mathrm{~mm}^{2} / \mathrm{s}$. These images were acquired with an acquisition period of 1-2 min only.

Images were transmitted to a workstation, and qualitative and quantitative assessments of DW-ADC maps were done. We did a qualitative assessment of DW-ADC maps by visually comparing the diffusion-weighted images with ADC maps to look for the presence or absence of diffusion restriction in pathological vertebrae. Vertebrae showing a high signal on diffusion images and corresponding low signal intensity on ADC maps were considered as a true diffusion restriction.

Table 1. Showing details of the magnetic resonance imaging protocol used in this study

\begin{tabular}{|l|c|c|c|c|c|c|c|}
\hline SEQ & $\begin{array}{c}\text { FOV/RFOV } \\
(\%)\end{array}$ & Matrix & $\begin{array}{c}\text { Thickness } \\
(\mathrm{mm})\end{array}$ & $\begin{array}{c}\text { GAP } \\
(\mathrm{mm})\end{array}$ & NSA & TR & TE \\
\hline $\begin{array}{l}\text { T1W SE } \\
\text { (sagittal) }\end{array}$ & $300 / 100$ & $216 / 512$ & 4.0 & 0.4 & 2 & $400-500$ & $14-16$ \\
\hline $\begin{array}{l}\text { T2W TSE } \\
\text { (axial) }\end{array}$ & $200 / 90$ & $171 / 512$ & 4.0 & 0.4 & 3 & 4500 & 120 \\
\hline $\begin{array}{l}\text { T2W TSE } \\
\text { (sagittal) }\end{array}$ & $300 / 100$ & $221 / 512$ & 4.0 & 0.4 & 2 & 4500 & 120 \\
\hline $\begin{array}{l}\text { STIR } \\
\text { (sagittal) }\end{array}$ & $300 / 100$ & $216 / 152$ & 4.0 & 0.4 & 3 & 4000 & 60 \\
\hline $\begin{array}{l}\text { DWI } \\
\text { (sagittal) }\end{array}$ & $300 / 100$ & $89 / 128$ & 4.0 & 0.4 & 6 & 1750 & 85 \\
\hline $\begin{array}{l}\text { T1W (post contrast) } \\
\text { (sagittal) }\end{array}$ & $300 / 100$ & $216 / 512$ & 4.0 & 0.4 & 3 & 500 & 20 \\
\hline
\end{tabular}


For quantitative analysis, the most solid and uniform parts of the lesion as seen on T2-weighted and contrastenhanced images were chosen for measurement. A round region of interest (ROI) was positioned on the area of interest, and the mean ADC values were computed. In case the lesion showed heterogeneous signal intensity, we used several small (at least three) identical round ROIs (area: smallest $10 \mathrm{~mm}^{2} /$ largest $55 \mathrm{~mm}^{2}$ ) that were positioned on the ADC map. We took care that the ROI encompassed the areas of contrast enhancement along with the lowermost ADC values (hypointensity) on visual inspection. The ROI location was confirmed by correlating it with corresponding conventional MRI images to circumvent contamination from adjacent structures.

The mean ADC value was calculated, and each lesion was predicted as benign or malignant based on its conventional MRI characteristics and ADC values.

\section{Standard of reference}

The diagnosis of all the malignant vertebral lesions was confirmed on histopathological examination after procuring the sample with computed tomography (CT)-guided bone biopsy. The diagnosis of infectious spondylitis was made with the aid of CT-guided bone biopsy in 18 patients and by the combination of clinical features, laboratory findings, and MRI follow-up in 15 patients.

The corroborating laboratory findings comprised high levels of C-reactive protein (CRP), raised erythrocyte sedimentation rate (ESR), raised leucocyte count, and positive blood cultures. Osteoporosis was identified by bone densitometry with a T-score of -2.5 or lower [7], and the diagnosis of all the vertebral haemangiomas was made by corroborating MRI findings with findings on other imaging modalities, i.e. by a parallel striated pattern (corduroy cloth) on plain film or a spotted appearance (polka dot) on axial CT images [8].

All patients were followed up clinically and by MRI for at least six months, and the diagnosis was confirmed in all cases.

\section{Statistical analysis}

The statistical examination was done with the Statistical Package for the Social Sciences for Windows (SPSS) software. Microsoft Word and Excel were used to form graphs, tables, and flowcharts. We used the unpaired $t$-test to assess our hypothesis that quantitative DWI can distinguish between benign and malignant vertebral pathologies. $P<0.05$ was deemed as the statistical significance level. The threshold ADC measurement to differentiate the benign group from the malignant group was acquired by receiver operating characteristic (ROC) analysis. Sensitivity, specificity, and positive and negative predictive values were estimated by using this cut-off value.

\section{Results}

A total of 302 vertebral lesions were seen in 100 patients, of whom 60 (60\%) were males and 40 (40\%) were females with ages ranging from 7 to 88 years and with a mean age of 53.47 years. Out of a total of 302 vertebral lesions, maximum vertebral lesions were seen in the lumbar $(n=138$, $45.69 \%$ ), which was followed by thoracic ( $n=130,43.04 \%)$ and sacral regions $(n=34,11.25 \%)$. L4 was the most frequently involved vertebra $(n=65,21.7 \%)$ that was followed by L1 $(n=61,20.2 \%)$. Vertebral bone marrow metastases were most common from breast cancer in females and prostate cancer in males. Vertebral pathologies were divided into three groups according to aetiology. Group 1 included benign (non-infections) lesions $(n=32)$, group 2 comprised infectious spondylodiscitis $(n=33)$, and group 3 included malignant lesions $(n=35)$ (Table 2$)$.

\section{The role of conventional magnetic resonance imaging sequences}

Out of 100 cases, conventional MRI sequences predicted 56 out of 58 benign lesions accurately as benign and 33 out of 42 lesions as malignant, with sensitivity, specificity, and positive predictive and negative predictive values of $94.29 \%, 86.15 \%, 78.57 \%$, and $91.67 \%$, respectively, i.e. benign lesions were more accurately diagnosed. The $p$-value was $<0.05$, which proved a significant correlation between the conventional MRI findings and the actual nature of lesions found on histopathology/clinical follow-up/other investigations.

\section{Qualitative analysis of diffusion-weighted magnetic resonance imaging}

All the benign vertebral lesions (benign collapse, haemangiomas, and infectious spondylodiscitis) were hypointense on T1 W, and hyperintense on T2 W and STIR sequences. On DWI (with respect to the signal intensity of normal bone marrow), out of 32 acute benign lesions (benign collapse and haemangiomas), 28 (87.5\%) were

Table 2. Distribution of various bone marrow lesions

\begin{tabular}{|c|c|c|}
\hline Variable & Number (n) & Percentage $(\%)$ \\
\hline \multicolumn{3}{|l|}{ Benign lesions } \\
\hline Haemangioma & 16 & 16 \\
\hline Benign collapse & 16 & 16 \\
\hline \multicolumn{3}{|l|}{ Infections } \\
\hline Pyogenic & 13 & 13 \\
\hline Tubercular & 20 & 20 \\
\hline \multicolumn{3}{|l|}{ Malignant } \\
\hline Metastasis & 24 & 24 \\
\hline Primary neoplasm & 11 & 11 \\
\hline
\end{tabular}




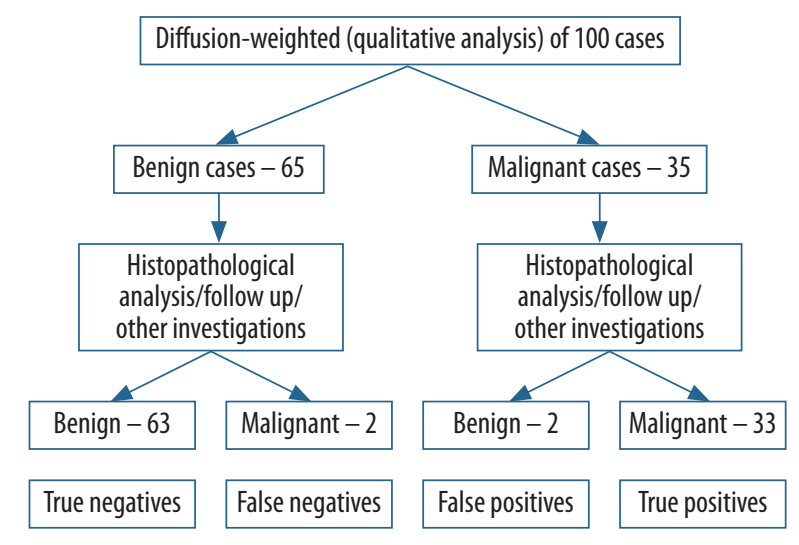

Figure 1. Qualitative diffusion-weighted analysis of the vertebral lesions

hypointense and four (12.5\%) were isointense. Amongst the infectious group, hyperintense signal was seen in two (10\%) out of 20 tuberculous lesions. In cases of pyogenic spondylodiscitis, hypointense signal on DWI was seen in all 13 lesions. Hyperintense signal on DW-MRI images was observed in all (100\%) malignant lesions. Out of 100 cases, we categorised the bone marrow lesions into benign and malignant based on the signal intensity on DWI along with the corresponding signal from ADC maps and conventional sequences. We were able to predict 63 out of 65 lesions correctly as benign and 33 out of 35 lesions correctly as malignant. DWI-ADC maps when analysed with corresponding conventional MRI sequences were $94.29 \%$ sensitive and $96.92 \%$ specific in predicting the malignant nature of the lesion in visual analysis (Figure 1).

\section{Quantitative analysis of diffusion-weighted magnetic} resonance imaging - apparent diffusion coefficient value

The mean $\mathrm{ADC}$ value $\left( \pm \mathrm{SD} \times 10^{-3} \mathrm{~mm}^{2} / \mathrm{s}\right)$ of normal vertebrae was computed as $0.39 \pm 0.13 \times 10^{-3} \mathrm{~mm}^{2} / \mathrm{s}$, and no statistically significant differences were found regarding the mean $\mathrm{ADC}$ values of normal vertebrae among the patient groups ( $p=0.705, p>0.05$ ). The mean ADC value of diseased vertebrae was $1.32 \pm 0.54 \times 10^{-3} \mathrm{~mm}^{2} / \mathrm{s}$, and a statisti- cally significant difference was observed amongst the mean ADC values of the normal and diseased vertebrae $(p<0.05)$ (Table 2). The mean ADC value of the benign pathologies (benign compression fractures and infections) was $1.66 \pm 0.32 \times 10^{-3} \mathrm{~mm}^{2} / \mathrm{s}$ and malignant lesions were $0.69 \pm 0.16 \times 10^{-3} \mathrm{~mm}^{2} / \mathrm{s}$, and this difference was statistically significant $(p<0.05)$. A threshold ADC value was calculated using the receiver operating characteristic curve (ROC curve) to separate benign from malignant pathologies. The threshold value for the mean ADC value was found to be $1.21 \times 10^{-3} \mathrm{~mm}^{2} / \mathrm{s}$. According to the above optimal threshold value established to discern vertebral bone marrow lesions, out of 100 cases, we predicted 60 out of 65 lesions correctly as benign and all 35 malignant lesions correctly. Therefore, ADC was found to be $100 \%$ sensitive and $92.31 \%$ specific in predicting the malignant nature of the lesions. The $p$-value was $<0.001$, which proved a highly significant correlation between findings predicted by ADC and the actual nature of lesions found in histopathology/ clinical follow-up/other investigations (Tables 3 and 4, Figure 2).

\section{Discussion}

Over the last decade, DW-MRI of the spine has proven its value and has been successfully employed in many clinical situations [9]. In the present study, 100 patients with 302 vertebral lesions were recruited. L4 was the most frequently involved vertebra, and the second most frequently involved vertebra was L1. These findings were akin to results documented by some previous studies [10,11].

In the current study, qualitative DWI evaluation of pathological vertebrae revealed that, out of 32 acute benign lesions (benign collapse and haemangiomas), 28 (87.5\%) were hypointense and four (12.5\%) were isointense. The osteoporotic compression fractures tend to show diminished signal on DWI due to the free diffusivity of water (oedema) between the interstices of bone [12]. Among the infectious group, the hyperintense signal on DWI was observed in two (10\%) out of 20 tuberculous lesions. In pyogenic spondylodiscitis, the hypointense signal was ob-

Table 3. Showing apparent diffusion coefficient (ADC) values in different benign and malignant pathologies

\begin{tabular}{|c|c|c|c|c|}
\hline \multirow[t]{2}{*}{ Lesions } & \multirow[t]{2}{*}{ Number } & \multicolumn{2}{|c|}{ Mean ADC value (in $\mathrm{mm}^{2} / \mathrm{sec}$ ) } & \multirow[t]{2}{*}{$P$-value } \\
\hline & & Normal & Diseased & \\
\hline \multicolumn{5}{|l|}{ Benign } \\
\hline Haemangiomas & 16 & $0.36 \pm 0.13 \times 10^{-3}$ & $1.82 \pm 0.17 \times 10^{-3}$ & 0.0001 \\
\hline Benign collapse & 16 & $0.42 \pm 0.12 \times 10^{-3}$ & $1.94 \pm 0.18 \times 10^{-3}$ & 0.0001 \\
\hline \multicolumn{5}{|l|}{ Infections } \\
\hline Pyogenic & 13 & $0.40 \pm 0.11 \times 10^{-3}$ & $1.47 \pm 0.22 \times 10^{-3}$ & 0.0001 \\
\hline Tuberculosis & 20 & $0.37 \pm 0.14 \times 10^{-3}$ & $1.42 \pm 0.31 \times 10^{-3}$ & 0.0001 \\
\hline \multicolumn{5}{|l|}{ MALIGNANT } \\
\hline Metastases & 24 & $0.39 \pm 0.14 \times 10^{-3}$ & $0.69 \pm 0.18 \times 10^{-3}$ & 0.0001 \\
\hline Primary neoplasm & 11 & $0.41 \pm 0.11 \times 10^{-3}$ & $0.69 \pm 0.12 \times 10^{-3}$ & 0.0001 \\
\hline
\end{tabular}


served in all 13 lesions. Our outcomes were akin to what has been documented in some previous studies. However, there is a disagreement in the literature $[6,12-16]$ on the role of DWI in distinguishing benign and malignant vertebral fractures, which might be due to use of the lower $b$-values and dissimilar sequences by previous authors and due to complexity with the overlap of oedema, haematoma, and bone fragmentation in both conditions. As a result, to date, there is no definitive consensus on DWI as the definitive tool for the distinction of benign fractures from pathological fractures [12]. Nevertheless, we discovered that DWI adds sensitivity to the detection of osseous pathologies of the spine. In addition to the usual sequences used for evaluation of questionable malignant lesions, DWI increases the conspicuity of several lesions. In some recent studies it has been documented that malignant lesions were more obvious when DWI was combined with other routine MRI sequences, which was also observed in our study [6].

In the current analysis, the mean $\mathrm{ADC}$ measurement of normal vertebrae was $0.39 \pm 0.13 \times 10^{-3} \mathrm{~mm}^{2} / \mathrm{s}$, and the mean ADC measurement of all the pathological vertebrae (benign, infectious and malignant) was 1.32 $\pm 0.54 \times 10^{-3} \mathrm{~mm}^{2} / \mathrm{s}$, which was significantly greater than that of normal ones $(p<0.0001)$, and this finding is indicative of diminished diffusivity of the normal fatty marrow. The tumour cells infiltrate and replace the bone marrow, thus also limiting free water diffusion [6]. Accordingly, we found that the mean ADC measurement of vertebrae with malignant marrow lesions was less than those with benign pathologies. However, the mean ADC value of the vertebrae with malignant marrow pathologies was greater than those with normal fatty marrow (Figure 3). On the other hand, benign acute vertebral fractures have an increased quantity of free water in the interstitial region with an escalation of water diffusion $[17,18]$ (Figure 4) and leading to high ADC values. In the present analysis, we observed that the mean $\mathrm{ADC}$ value of benign pathologies $\left(1.66 \pm 0.32 \times 10^{-3} \mathrm{~mm}^{2} / \mathrm{s}\right)$ was statistically greater than those of malignant ones $(0.69$ $\left.\pm 0.15 \times 10^{-3} \mathrm{~mm}^{2} / \mathrm{s}\right)(p<0.0001)$. Therefore, diffusivity (ADC values) was found to be highest in benign lesions, followed by malignant pathologies, and least in vertebrae with normal fatty marrow. Our outcomes are in agreement with the many previous studies in the literature [6]. In research performed by Wonglaksanapimon et al. [19], seven malignant fractures and 32 benign fractures were assessed. Similarly to our study, the disparity amongst the ADC measurements of the benign and malignant fractures was statistically significant $(p<0.0001)$.

In consonance with the literature, we found a significant variance amongst the ADC values of benign and malignant fractures with a lack of overlap [20-22]. In contrast to our observations, Turna et al. documented that ADC values were not useful in distinguishing benign and malignant compression fractures, and there was an overlap
Table 4. Showing cut-off apparent diffusion coefficient (ADC) value and $A D C$ values in different vertebral lesions

\begin{tabular}{|l|c|c|}
\hline Cut-off value for mean ADC & $\begin{array}{c}\text { No. of malignant } \\
\text { cases }\end{array}$ & $\begin{array}{c}\text { No. of benign } \\
\text { cases }\end{array}$ \\
\hline $1.21 \times 10^{-3} \mathrm{~mm}^{2} / \mathrm{s}$ or lower & 35 & 5 \\
\hline Over $1.21 \times 10^{-3} \mathrm{~mm}^{2} / \mathrm{s}$ & 0 & 60 \\
\hline Total & 35 & 65 \\
\hline
\end{tabular}

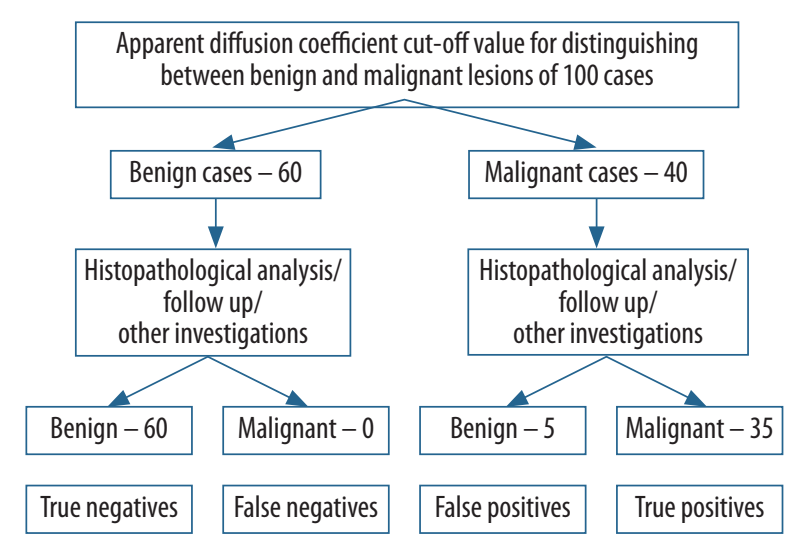

Figure 2. Quantitative diffusion-weighted analysis of the vertebral lesions

of ADC values [10]. This disagreement might be linked to technical variations amongst studies as well as dissimilar stages of the diseases during the studies.

In the present study, the mean ADC measurement of the lesions due to pyogenic infections was $1.47 \pm 0.22$ $\times 10^{-3} \mathrm{~mm}^{2} / \mathrm{s}$ and was significantly greater than that of malignant ones $\left(0.69 \pm 0.15 \times 10^{-3} \mathrm{~mm}^{2} / \mathrm{s}\right)(p<0.05)$ with an absence of overlap. In agreement with the present study, many studies in the literature [6] have also documented that the mean ADC values of infectious lesions are significantly greater than malignant lesions $(p<0.05)$. We observed that only two of the 23 vertebral infectious lesions displayed $\mathrm{ADC}$ values overlapping with the malignant ones (Figure 5). Similarly to our results, some studies $[20,23]$ have documented that the ADC measurements of infectious and malignant pathologies overlapped and hence could not be used to differentiate these entities. Different stages of infection may show different ADC values, which may vary with the stage of infection, and we feel that long follow-up studies are required to know the exact variation of ADC values with time in the cases having vertebral infections. Therefore, considering the literature, distinction of infectious and malignant pathologies by ADC measurements can be unpredictable. We observed that the mean ADC measurement of the five tuberculous lesions was $0.91 \pm 0.08 \times 10^{-3} \mathrm{~mm}^{2} / \mathrm{s}$ with a non-significant difference compared to the values of malignant pathologies $(p=0.143)$, and there was an overlap of ADC values between tuberculous and malignant lesions. This may be due to dense solid caseation within the tubercular vertebrae, so the overlap with ADC measurements of malignant 

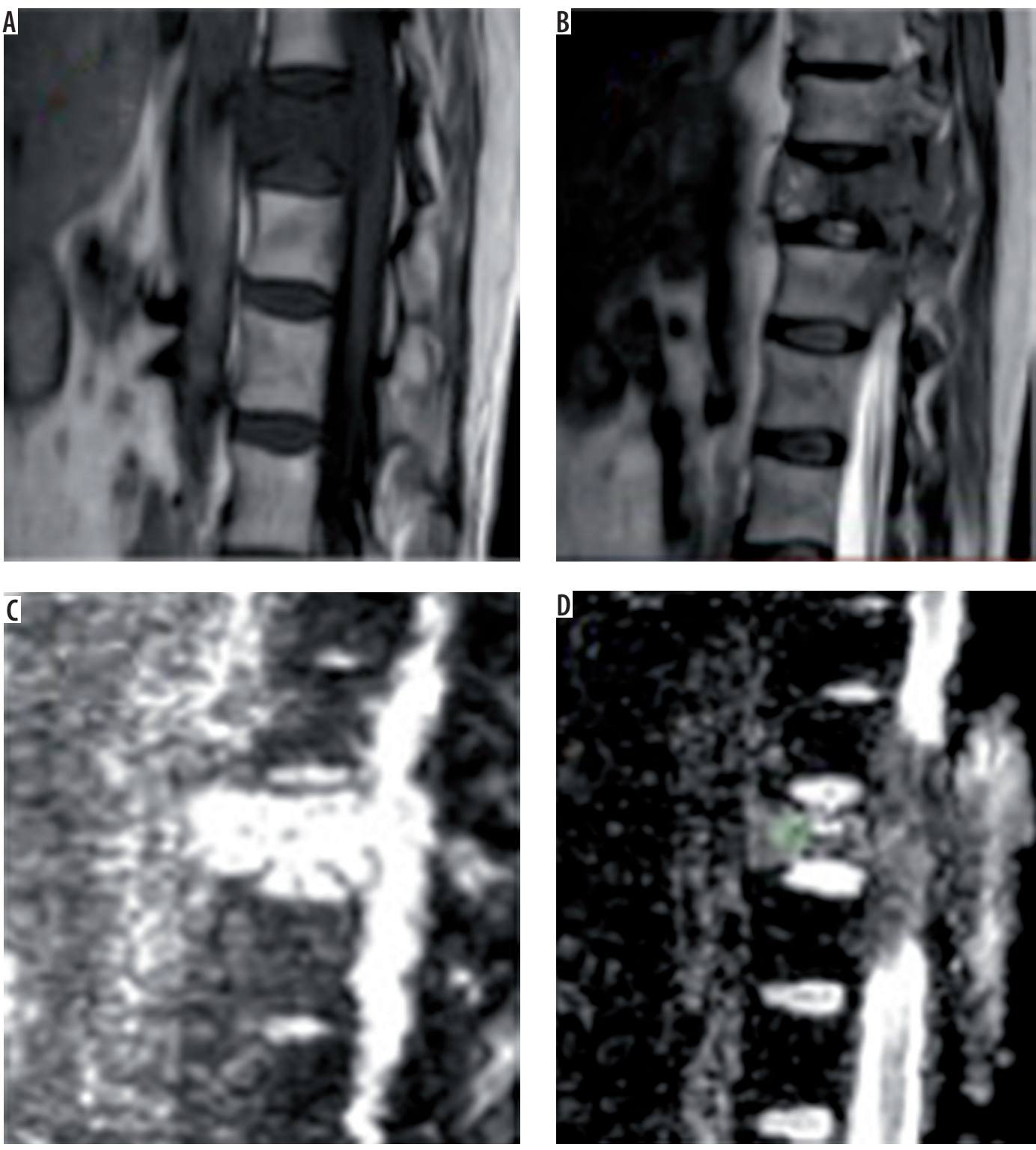

Figure 3. In a known case of breast cancer, 50-year-old female presented with gradual onset backache. A, B) (sagittal T1WI \& T2WI) show evidence of altered marrow signal intensity in D11 vertebral body, pedicles and laminae appearing hypointense on T1WI and heterogeneously hyperintense on T2WI. Epidural soft tissue is seen at this level compressing the cord. Area of altered marrow signal intensity is also observed involving posterior aspect of D12 vertebral body. C, D) (DWI \& ADC map) on diffusion-weighted image lesion display high signal intensity (SI). The mean ADC value was $(0.8 \pm 0.02$ $\times 10^{-3} \mathrm{~mm}^{2} / \mathrm{s}$ ) coinciding with malignant nature. On biopsy, it proved to be malignant

lesions may be noted. Palle et al. [24] also documented the mean ADC measurements of 128 vertebral tuberculosis lesions in 56 cases to be $1.4 \times 10^{-3} \mathrm{~mm}^{2} / \mathrm{s}$, and after considering this measurement as a threshold to differentiate infective from malignant lesions, they observed $64.8 \%$ sensitivity, $75 \%$ specificity, and $74.5 \%$ positive predictive values, and there was an overlap of ADC values. These outcomes are comparable to ours, but the number of tuberculosis lesions in our study was less than in the above-cited study.

In the present analysis, we were able to discern malignant and benign pathologies on quantitative DW-ADC maps with good sensitivity, specificity, positive predictive value, and negative predictive value using a threshold value of $1.21 \times 10^{-3} \mathrm{~mm}^{2} / \mathrm{s}$. Therefore, we believe that DWI will emerge as an accurate marrow evaluation sequence for detection and assessment of therapy of metastases involving the vertebrae. The major advantage of this sequence is that no ionising radiation is administered and no injection of isotopes or any contrast medium is required. Short data acquisition time is another advantage. Therapy assessment may be done by observing changes in extent, symmetry, and intensity of signal on high $b$ values and corresponding alterations in ADC values is another exciting area of future research. Furthermore, we firmly feel that DWI can have a significant impact on the management of patients with malignant diseases in the near future. The main drawback of our study is the small sample size and short follow-up, so further studies with larger series and with a longer follow-up are required to 

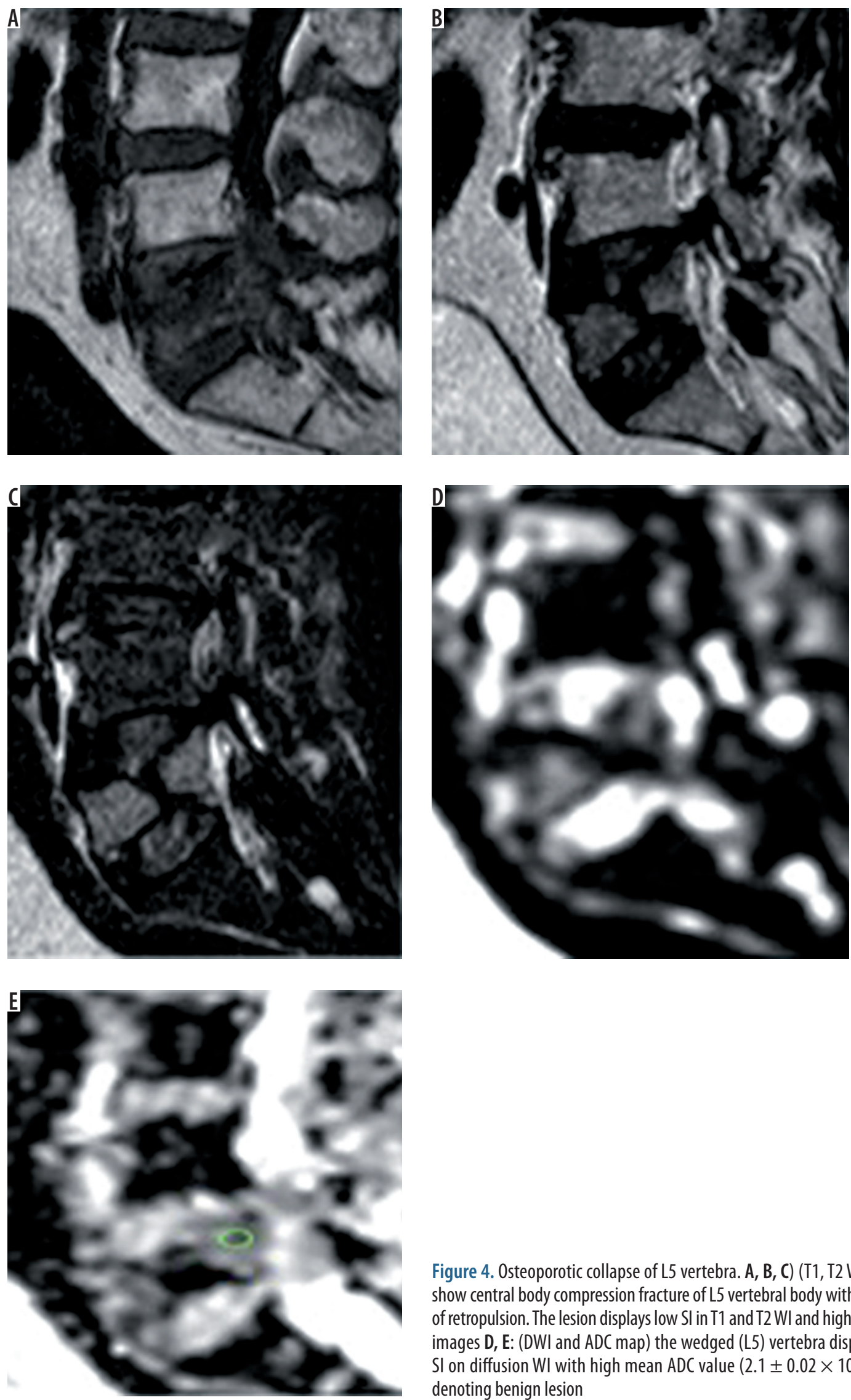

Figure 4. Osteoporotic collapse of L5 vertebra. A, B, C) (T1, T2 WI \& STIR) show central body compression fracture of $L 5$ vertebral body with evidence of retropulsion. The lesion displays low SI in T1 and T2 WI and high SI on STIR images D, E: (DWI and ADC map) the wedged (L5) vertebra displays high $\mathrm{SI}$ on diffusion WI with high mean ADC value $\left(2.1 \pm 0.02 \times 10^{-3} \mathrm{~mm}^{2} / \mathrm{s}\right)$ denoting benign lesion

see changes in the $\mathrm{ADC}$ values during the disease process to confirm these results. Therapy response criteria of DWI ought to be established so that they can then be examined in prospective investigations. 

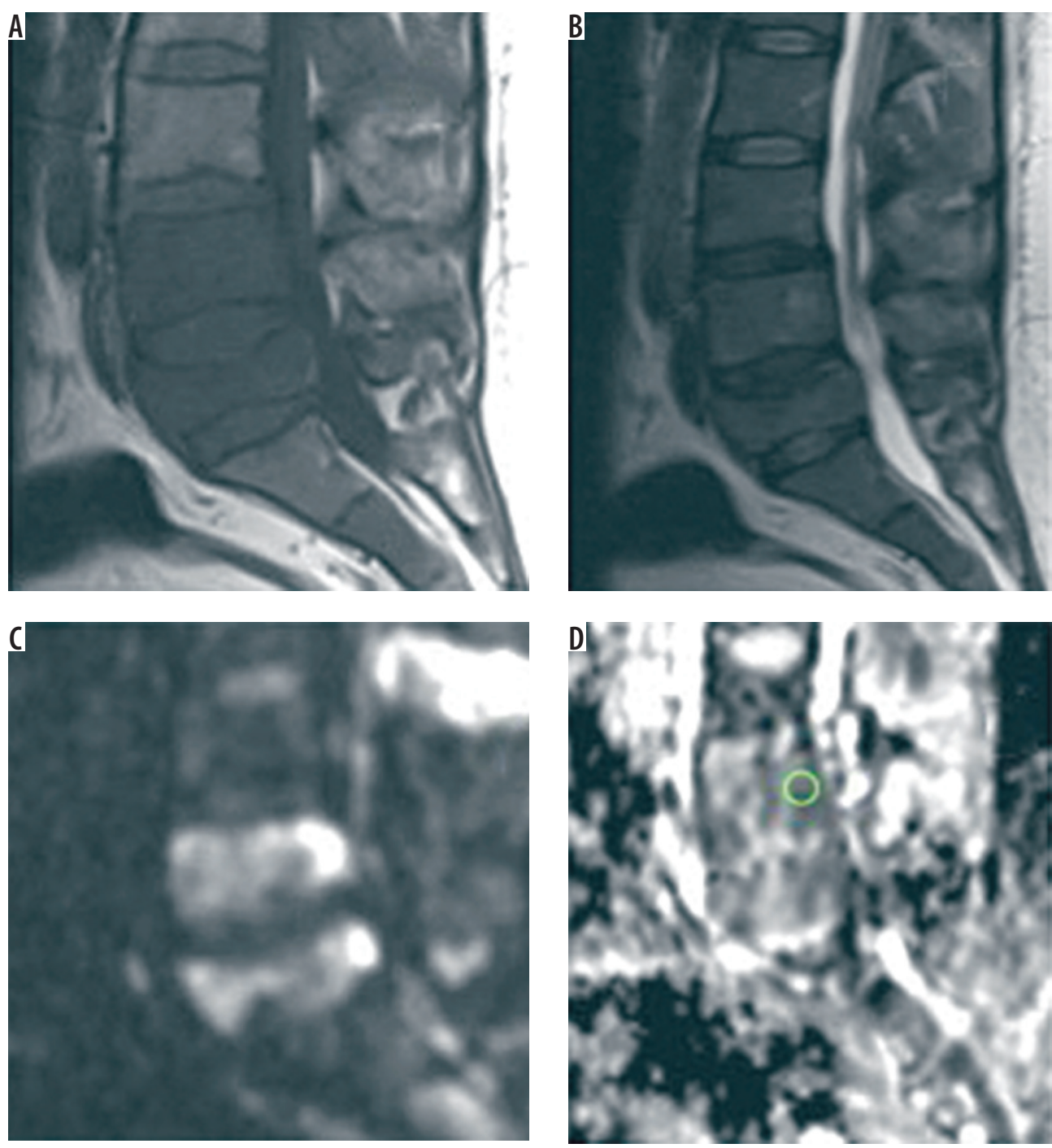

Figure 5. A case of lymphoma. A, B) (T1W, T2 W) showing vertebral lymphomatous infiltrations with the collapse of L5 vertebra displaying low SI on T1 and isointense SI in T2-weighted images. C, D: (DWI and ADC map). On diffusion WI the lesion shows high SI with mean ADC value $\left(1.0 \pm 0.02 \times 10^{-3} \mathrm{~mm}^{2} / \mathrm{s}\right)$

\section{Conclusions}

DW-MRI of the vertebral marrow is a beneficial sequence that can be used for the categorisation of bone-marrow pathologies. Quantitative ADC values offer useful information in distinguishing benign lesions from malignant disorders. Hence, DW-ADC maps should be acquired routinely to segregate vertebral marrow pathologies because ADC values have good sensitivity and specificity for the same.

\section{Conflict of interest}

The authors report no conflict of interest.

\section{References}

1. Leake RL, Mills MK, Hanrahan CJ. Spinal marrow imaging: clues to disease. Radiol Clin North Am 2019; 57: 359-375.

2. Lin S, Ouyang T, Kanekar S. Imaging of bone marrow. Hematol Oncol Clin North Am 2016; 30: 945-971.

3. Schieda N, Blaichman JI, Costa AF, et al. Gadolinium-based contrast agents in kidney disease: a comprehensive review and clinical practice guideline issued by the Canadian Association of Radiologists. Can J Kidney Health Dis 2018; 5: 20543581 18778573.
4. Dietrich O, Geith T, Reiser MF, Baur-Melnyk A. Diffusion imaging of the vertebral bone marrow. NMR Biomed 2017; 30: e3333.

5. Karampinos DC, Ruschke S, Dieckmeyer M, et al. Quantitative MRI and spectroscopy of bone marrow. J Magn Reson Imaging 2018; 47: 332-353.

6. Suh $\mathrm{CH}$, Yun SJ, Jin W, et al. ADC as a useful diagnostic tool for differentiating benign and malignant vertebral bone marrow lesions and compression fractures: a systematic review and meta-analysis. Eur Radiol 2018; 28: 2890-2992. 
7. Sheu A, Diamond T. Bone mineral density: testing for osteoporosis. Aust Prescr 2016; 39: 35-39.

8. Mahajan PS, Jayaram AP, Negi VC. Rare case of multiple aggressive vertebral hemangiomas. J Nat Sci Biol Med 2015; 6: 439-442.

9. Drake-Pérez M, Boto J, Fitsiori A, et al. Clinical applications of diffusion weighted imaging in neuroradiology. Insights Imaging 2018; 9: 535-547.

10. Turna O, Aybar MD, Tuzcu G, et al. Evaluation of vertebral bone marrow with diffusion weighted MRI and ADC measurements. Istanbul Med J 2014; 15: 116-121.

11. Abo Dewan KAW, Salama AA, El Habashy HMS, et al. Evaluation of benign and malignant vertebral lesions with diffusion weighted magnetic resonance imaging and apparent diffusion coefficient measurements. Egypt J Radiol Nucl Med 2015; 46: 423-433.

12. Mauch JT, Carr CM, Cloft H, et al. Review of the Imaging Features of Benign Osteoporotic and Malignant Vertebral Compression Fractures. AJNR Am J Neuroradiol 2018; 39: 1584-1592.

13. Moritani T, Kim J, Capizzano AA, et al. Pyogenic and non-pyogenic spinal infections: emphasis on diffusion-weighted imaging for the detection of abscesses and pus collections. Br J Radiol 2014; 87: 20140011.

14. Castillo M, Arbelaez A, Smith JK, et al. Diffusion-weighted MR imaging offers no advantage over routine noncontrast MR imaging in the detection of vertebral metastases. AJNR Am J Neuroradiol 2000; 21: 948-953.

15. Luo Z, Litao L, Gu S, et al. Standard-b-value vs low-b-value DWI for differentiation of benign and malignant vertebral fractures: a metaanalysis. Br J Radiol 2016; 89: 20150384.

16. Zhang SW, Xu L, Zhong TK, et al. Comments on "Standard-b-value vs low-b-value DWI for differentiation of benign and malignant vertebral fractures: a meta-analysis". Br J Radiol 2016; 89: 20160224.
17. Baur A, Huber A, Ertl-Wagner B, et al. Diagnostic value of increased diffusion weighting of a steady-state free precession sequence for differentiating acute benign osteoporotic fractures from pathologic vertebral compression fractures. AJNR Am J Neuroradiol 2001; 22: 366-372.

18. Padhani AR, Koh DM, Collins DJ. Whole-body diffusion-weighted MR imaging in cancer: current status and research directions. Radiology 2011; 261: 700-718.

19. Wonglaksanapimon S, Chawalparit O, Khumpunnip S, et al. Vertebral body compression fracture: discriminating benign from malignant causes by diffusion-weighted MR imaging and apparent diffusion coefficient value. J Med Assoc Thai 2012; 95: 81-87.

20. Balliu E, Vilanova JC, Peláez I, et al. Diagnostic value of apparent diffusion coefficients to differentiate benign from malignant vertebral bone marrow lesions. Eur J Radiol 2009; 69: 560-566.

21. Baur A, Stäbler A, Brüning R, et al. Diffusion-weighted MR imaging of bone marrow: differentiation of benign versus pathologic compression fractures. Radiology 1998; 207: 349-356.

22. Bhugaloo A, Abdullah B, Siow Y, et al. Diffusion weighted MR imaging in acute vertebral compression fractures: differentiation between malignant and benign causes. Biomed Imaging Interv J 2006; 2: e12.

23. Pui MH, Mitha A, Rae WI, Corr P. Diffusion-weighted magnetic resonance imaging of spinal infection and malignancy. J Neuroimaging 2005; 15: 164-170.

24. Palle L, Reddy MB, Reddy KJ. Role of magnetic resonance diffusion imaging and apparent diffusion coefficient values in the evaluation of spinal tuberculosis in Indian patients. Indian J Radiol Imaging 2010; 20: 279-283. 